\title{
MAPS PRESERVING THE LOCAL SPECTRUM OF PRODUCT OF OPERATORS
}

\author{
ABDELLATIF BOURHIM \\ Department of Mathematics, Syracuse University, \\ 215 Carnegie Building, Syracuse, NY 13244, USA \\ e-mail: abourhim@syr.edu \\ and JAVAD MASHREGHI \\ Département de mathématiques et de statistique, Université Laval, \\ Québec, QC, G1V0A6, Canada \\ e-mail: javad.mashreghi@mat.ulaval.ca
}

(Received 26 February 2014; accepted 13 March 2014; first published online 17 December 2014)

\begin{abstract}
Let $X$ and $Y$ be infinite-dimensional complex Banach spaces, and $\mathcal{B}(X)$ (resp. $\mathcal{B}(Y)$ ) be the algebra of all bounded linear operators on $X$ (resp. on $Y$ ). For an operator $T \in \mathcal{B}(X)$ and a vector $x \in X$, let $\sigma_{T}(x)$ denote the local spectrum of $T$ at $x$. For two nonzero vectors $x_{0} \in X$ and $y_{0} \in Y$, we show that a map $\varphi$ from $\mathcal{B}(X)$ onto $\mathcal{B}(Y)$ satisfies

$$
\sigma_{\varphi(T) \varphi(S)}\left(y_{0}\right)=\sigma_{T S}\left(x_{0}\right),(T, S \in \mathcal{B}(X)),
$$

if and only if there exists a bijective bounded linear mapping $A$ from $X$ into $Y$ such that $A x_{0}=y_{0}$ and either $\varphi(T)=A T A^{-1}$ or $\varphi(T)=-A T A^{-1}$ for all $T \in \mathcal{B}(X)$.

2000 Mathematics Subject Classification. Primary 47B49; Secondary 47A10, 47A11.
\end{abstract}

1. Introduction. Throughout this paper, $X$ and $Y$ denote infinite-dimensional complex Banach spaces, and $\mathcal{B}(X, Y)$ denotes the space of all bounded linear maps from $X$ into $Y$. When $X=Y$, we simply write $\mathcal{B}(X)$ instead of $\mathcal{B}(X, X)$. The local resolvent set, $\rho_{T}(x)$, of an operator $T \in \mathcal{B}(X)$ at a point $x \in X$ is the union of all open subsets $U$ of $\mathbb{C}$ for which there is an analytic function $\phi: U \rightarrow X$ such that $(T-\lambda) \phi(\lambda)=$ $x, \quad(\lambda \in U)$. The local spectrum of $T$ at $x$ is defined by $\sigma_{T}(x):=\mathbb{C} \backslash \rho_{T}(x)$, and is obviously a closed subset of $\sigma(T)$, the spectrum of $T$. The local spectral radius of $T$ at $x$ is defined by $r_{T}(x):=\lim \sup _{n \rightarrow+\infty}\left\|T^{n} x\right\|^{\frac{1}{n}}$, and coincides with maximum modulus of $\sigma_{T}(x)$ provided that $T$ has the single-valued extension property (SVEP). Recall that $T \in \mathcal{B}(X)$ is said to have SVEP provided that for every open subset $U$ of $\mathbb{C}$, the equation $(T-\lambda) \phi(\lambda)=0, \quad(\lambda \in U)$ has no nontrivial analytic solution $\phi$. Every operator $T \in \mathcal{B}(X)$ for which the interior of its point spectrum, $\sigma_{p}(T)$, is empty enjoys this property. The remarkable books by P. Aiena [1] and by K.B. Laursen, M.M. Neumann [13] provide an excellent exposition as well as a rich bibliography of the local spectral theory.

Several results on linear or additive preserver problems have been extended to the setting of nonlinear preservers, and, in many cases, their extensions demonstrated to be nontrivial. In particular, the problem of characterizing maps preserving certain 
functions, subsets, relations and properties of product of matrices or operators has attracted the attention of several authors; see for instance $[2,7,10,11,15,14,17]$ and the references therein. Motivated by problems concerning local automorphisms L. Molnár characterized, in [15], maps preserving different spectra of operator or matrix products. In [11], Hou and Di described, in particular, maps preserving the numerical range of products of Hilbert space operators. In [14], maps preserving the nilpotency of operator products are characterized.

On the other hand, the problem of describing linear or additive maps on $\mathcal{B}(X)$ preserving the local spectra has been initiated by A. Bourhim and T. Ransford in [5], and continued by several authors; see for instance $[6,8,9]$ and the references therein. In [8], C. Costara described surjective linear maps on $\mathcal{B}(X)$ which preserve operators of local spectral radius zero at points of $X$. He showed, in particular, that if $\varphi$ is a surjective linear map on $\mathcal{B}(X)$ such that for every $x \in X$ and $T \in \mathcal{B}(X)$, we have $\mathrm{r}_{T}(x)=0$ if and only if $\mathrm{r}_{\varphi(T)}(x)=0$, then there exists a nonzero scalar $c$ such that $\varphi(T)=c T$ for all $T \in \mathcal{B}(X)$. This result has been extended in [3] to the nonlinear setting where it was shown that if $\varphi$ is a surjective (not necessarily linear) map on $\mathcal{B}(X)$ satisfying $\mathrm{r}_{T-S}(x)=0$ if and only if $\mathrm{r}_{\varphi(T)-\varphi(S)}(x)=0$, for every $x \in X$ and $S, T \in \mathcal{B}(X)$, then there is a nonzero scalar $c \in \mathbb{C}$ and an operator $A \in \mathcal{B}(X)$ such that $\varphi(T)=c T+A$ for all $T \in \mathcal{B}(X)$.

This paper is a continuation of our recent work on nonlinear preserver problems of local spectra of operators $[3,4]$, and the related works in [8]. It can be viewed as a step towards the study of problems on nonlinear preservers of local spectra. Specifically, we are interested in determining the structure of maps preserving the local spectrum at a fixed vector of product of operators. Our main result is the following theorem.

TheOrem 1.1. Let $x_{0} \in X$ and $y_{0} \in Y$ be two nonzero vectors. A map $\varphi$ from $\mathcal{B}(X)$ onto $\mathcal{B}(Y)$ satisfies

$$
\sigma_{\varphi(T) \varphi(S)}\left(y_{0}\right)=\sigma_{T S}\left(x_{0}\right),(T, S \in \mathcal{B}(X)),
$$

if and only if there exists a bijective bounded linear mapping $A$ from $X$ into $Y$ such that $A x_{0}=y_{0}$ and either $\varphi(T)=A T A^{-1}$ for all $T \in \mathcal{B}(X)$ or $\varphi(T)=-A T A^{-1}$ for all $T \in \mathcal{B}(X)$.

Our arguments are influenced by ideas from $[\mathbf{1 4}, \mathbf{1 5}]$ and the approach given therein, but the proof of the main result requires new ingredients such a new spectral characterization of rank one operators in term of the local spectrum. It also relies on a local spectral identity principle that characterizes in term of the local spectrum when two operators are the same. We also would like to point out that if $X$ and $Y$ are isomorphic Banach spaces, then the statements of our main result can be reduced to the case when $X=Y$ and $x_{0}=y_{0}$. But the fact that " $X$ and $Y$ are isomorphic" is a part of the conclusion of this result rather being a part of its hypothesis.

2. Background from local spectral theory and preliminaries. In this section, we fix some notion and gather together some useful lemmas needed for the proof of our main result. The first lemma summarizes some basic properties of the local spectrum which will be used frequently.

LeMma 2.1. For an operator $T \in \mathcal{B}(X)$, vectors $x, y \in X$ and a nonzero scalar $\alpha \in \mathbb{C}$, the following statements hold. 
(a) If T has SVEP, then $\sigma_{T}(x) \neq \emptyset$ provided that $x \neq 0$.

(b) $\sigma_{T}(\alpha x)=\sigma_{T}(x)$ if $\alpha \neq 0$, and $\sigma_{\alpha T}(x)=\alpha \sigma_{T}(x)$.

(c) $\sigma_{T}(x+y) \subset \sigma_{T}(x) \cup \sigma_{T}(y)$. The equality holds if $\sigma_{T}(x) \cap \sigma_{T}(y)=\emptyset$.

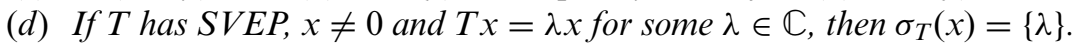

(e) If Thas SVEP and Tx $=\alpha y$, then $\sigma_{T}(y) \subset \sigma_{T}(x) \subset \sigma_{T}(y) \cup\{0\}$.

(f) If $R \in \mathcal{B}(X)$ commutes with $T$, then $\sigma_{T}(R x) \subset \sigma_{T}(x)$.

(g) $\sigma_{T^{n}}(x)=\left\{\sigma_{T}(x)\right\}^{n}$ for all $x \in X$ and $n \geq 1$.

Proof. See for instance $[1,13]$.

For any operator $T \in \mathcal{B}(X)$, let $T^{*}$ be its adjoint on the dual space $X^{*}$ of $X$. For every nonzero $x \in X$ and $f \in X^{*}$, we sometime write $\langle x, f\rangle$ instead $f(x)$ and let $x \otimes f$ denote the rank one operator defined on $X$ by $(x \otimes f)(y)=f(y) x, \sim(y \in X)$. Note that every rank one operator on $X$ can be written in this way and every finite rank operator is a finite sum of rank one operators. Let $\mathcal{F}(X)$ denote the ideal of all finite rank operators on $X$, and $\mathcal{F}_{n}(X)$ denote the set of all operators on $X$ of rank of at most $n$.

For a nonzero vector $x_{0} \in X$ and an operator $T \in \mathcal{B}(X)$, we use this notation

$$
\sigma_{T}^{*}\left(x_{0}\right):=\left\{\begin{array}{lll}
\{0\} & \text { if } & \sigma_{T}\left(x_{0}\right)=\{0\}, \\
\sigma_{T}\left(x_{0}\right) \backslash\{0\} & \text { if } & \sigma_{T}\left(x_{0}\right) \neq\{0\} .
\end{array}\right.
$$

The second lemma is a useful observation needed to establish the linearity of surjective maps $\varphi: \mathcal{B}(X) \rightarrow \mathcal{B}(Y)$ satisfying (1.1).

LemMa 2.2. Let $x_{0}$ be a nonzero vector in $X$. For two vectors $x$ and $y$ in $X$ and $a$ linear functional $f \in X^{*}$, the following statements hold.

(a)

$$
\sigma_{x \otimes f}^{*}\left(x_{0}\right)=\left\{\begin{array}{lll}
\{0\} & \text { if } & f\left(x_{0}\right)=0, \\
\{f(x)\} & \text { if } & f\left(x_{0}\right) \neq 0 .
\end{array}\right.
$$

(b) $\sigma_{(x+y) \otimes f}^{*}\left(x_{0}\right)=\sigma_{x \otimes f}^{*}\left(x_{0}\right)+\sigma_{y \otimes f}^{*}\left(x_{0}\right)$.

(c) For all rank one operators $R \in \mathcal{B}(X)$ and all $T, S \in \mathcal{B}(X)$, we have

$$
\sigma_{(T+S) R}^{*}\left(x_{0}\right)=\sigma_{T R}^{*}\left(x_{0}\right)+\sigma_{S R}^{*}\left(x_{0}\right) .
$$

Proof.

(a) If $f\left(x_{0}\right)=0$, then $(x \otimes f) x_{0}=0$ and $\sigma_{x \otimes f}\left(x_{0}\right)=\sigma_{x \otimes f}^{*}\left(x_{0}\right)=\{0\}$. If $f\left(x_{0}\right) \neq 0$, then $(x \otimes f) x_{0}=f\left(x_{0}\right) x$ and

$$
\{f(x)\}=\sigma_{x \otimes f}(x) \subset \sigma_{x \otimes f}\left(x_{0}\right) \subset \sigma_{x \otimes f}(x) \cup\{0\}=\{0, f(x)\} ;
$$

see Lemma 2.1-(e). It follows that $\sigma_{x \otimes f}^{*}\left(x_{0}\right)=\{f(x)\}$.

(b) If $f\left(x_{0}\right)=0$, then $\sigma_{(x+y) \otimes f}^{*}\left(x_{0}\right)=\sigma_{x \otimes f}^{*}\left(x_{0}\right)=\sigma_{y \otimes f}^{*}\left(x_{0}\right)=\{0\}$, and the desired identity holds. If $f\left(x_{0}\right) \neq 0$, then $\sigma_{(x+y) \otimes f}^{*}\left(x_{0}\right)=\{f(x+y)\}=\{f(x)\}+$ $\{f(y)\}=\sigma_{x \otimes f}^{*}\left(x_{0}\right)+\sigma_{y \otimes f}^{*}\left(x_{0}\right)$. 
(c) Write $R=x \otimes f$. Then, by part (b),

$$
\begin{aligned}
\sigma_{(T+S) R}^{*}\left(x_{0}\right) & =\sigma_{(T+S)(x \otimes f)}^{*}\left(x_{0}\right)=\sigma_{(T+S)(x) \otimes f}^{*}\left(x_{0}\right) \\
& =\sigma_{(T x+S x) \otimes f}^{*}\left(x_{0}\right)=\sigma_{T x \otimes f}^{*}\left(x_{0}\right)+\sigma_{S x \otimes f}^{*}\left(x_{0}\right) \\
& =\sigma_{T(x \otimes f)}^{*}\left(x_{0}\right)+\sigma_{S(x \otimes f)}^{*}\left(x_{0}\right)=\sigma_{T R}^{*}\left(x_{0}\right)+\sigma_{S R}^{*}\left(x_{0}\right) .
\end{aligned}
$$

LeMmA 2.3. Let $x_{0} \in X$ and $y_{0} \in Y$ be nonzero vectors, and let $A: X \rightarrow Y$ and $B: X^{*} \rightarrow Y^{*}$ be bijective linear transformations. The following statements are equivalent.

(a) For every $x \in X$ and $f \in X^{*}$, we have $\sigma_{x \otimes f}\left(x_{0}\right)=\sigma_{A x \otimes B f}\left(y_{0}\right)$.

(b) $A$ is continuous, $B=A^{*-1}$ and $A x_{0}=\alpha y_{0}$ for some nonzero scalar $\alpha \in \mathbb{C}$.

Proof. If $A$ is continuous, $B=A^{*-1}$ and $A x_{0}=\alpha y_{0}$ for some nonzero scalar $\alpha \in \mathbb{C}$, then $\sigma_{A x \otimes B f}\left(y_{0}\right)=\sigma_{A(x \otimes f) A^{-1}}\left(y_{0}\right)=\sigma_{x \otimes f}\left(A^{-1} y_{0}\right)=\sigma_{x \otimes f}\left(\alpha^{-1} x_{0}\right)=\sigma_{x \otimes f}\left(x_{0}\right)$ for all $x \in$ $X$ and $f \in X^{*}$. This establishes the implication $(b) \Rightarrow(a)$.

Conversely, assume that $\sigma_{x \otimes f}\left(x_{0}\right)=\sigma_{A x \otimes B f}\left(y_{0}\right)$ for all $x \in X$ and $f \in X^{*}$. Let $x \in X$ and $f \in X^{*}$, and let us show that

$$
\langle x, f\rangle=\langle A x, B f\rangle .
$$

Assume first that $f\left(x_{0}\right) \neq 0$, and note that, since $\sigma_{A x_{0} \otimes B f}\left(y_{0}\right)=\sigma_{x_{0} \otimes f}\left(x_{0}\right)=\left\{f\left(x_{0}\right)\right\}$, we infer that $B f\left(y_{0}\right) \neq 0$ and $A x_{0}=\alpha y_{0}$ for some nonzero $\alpha \in \mathbb{C}$. Since $\sigma_{x \otimes f}\left(x_{0}\right)=$ $\sigma_{A x \otimes B f}\left(y_{0}\right)$, we have $\{f(x)\}=\sigma_{x \otimes f}^{*}\left(x_{0}\right)=\sigma_{A x \otimes B f}^{*}\left(y_{0}\right)=\{B f(A x)\}$ and thus (2.2) holds.

Next consider the case when $f\left(x_{0}\right)=0$ and take a linear functional $g \in X^{*}$ such that $g\left(x_{0}\right) \neq 0$. It follows from what has been shown previously that $\langle x, g\rangle=\langle A x, B g\rangle$, and $\langle x,(f+g)\rangle=\langle A x, B(f+g)\rangle$. Expanding the last identity, we get $\langle x, f\rangle=\langle A x, B f\rangle$ which establishes (2.2) in this case too; as desired.

Now, let us show that $A$ is continuous and $B=A^{*-1}$. Assume that $\left(x_{n}\right)_{n} \subset X$ is such that $\lim _{n \rightarrow \infty} x_{n}=x \in X$ and $\lim _{n \rightarrow \infty} A x_{n}=y \in Y$. Then, for every $f \in X^{*}$, we have $\langle y, B f\rangle=\lim _{n \rightarrow \infty}\left\langle A x_{n}, B f\right\rangle=\lim _{n \rightarrow \infty}\left\langle x_{n}, f\right\rangle=\langle x, f\rangle=\langle A x, B f\rangle$. Since $B$ is bijective and $f \in X^{*}$ is an arbitrary linear functional, the closed graph theorem shows that $A$ is continuous.

Moreover, if $f \in X^{*}$ is a fixed linear functional, then for every $x \in X$, we have $\langle x, f\rangle=\langle A x, B f\rangle=\left\langle x, A^{*} B f\right\rangle$, and thus $A^{*} B=\mathbf{1}_{X^{*}}$. The proof is therefore complete.

LemmA 2.4. Let $x_{0} \in X$ and $y_{0} \in Y$ be nonzero vectors, and let $A: X^{*} \rightarrow Y$ and $B: X \rightarrow Y^{*}$ be bijective linear transformations. Then there are $x \in X$ and $f \in X^{*}$ such that $\sigma_{x \otimes f}\left(x_{0}\right) \neq \sigma_{A f \otimes B x}\left(y_{0}\right)$.

Proof. Assume by the way of contradiction that $\sigma_{x \otimes f}\left(x_{0}\right)=\sigma_{A f \otimes B x}\left(y_{0}\right)$ for all $x \in X$ and $f \in X^{*}$. Just as in the proof of the previous lemma, one can show that both $A$ and $B$ are continuous and thus both $X$ and $Y$ are reflexive. After identifying $X$ and $Y$ with their second dual spaces $X^{* *}$ and $Y^{* *}$, one can also show that $B=A^{*-1}$, and thus $\sigma_{R}\left(x_{0}\right)=\sigma_{A R^{*} A^{-1}}\left(y_{0}\right)=\sigma_{R^{*}}\left(A^{-1} y_{0}\right)$ for all rank one operators $R \in \mathcal{B}(X)$.

Let $x \in X$ be a nonzero element such that $x \notin \mathbb{C} x_{0}$ and $\left\langle x, A^{-1} y_{0}\right\rangle=1$, and let $f$ be a linear functional on $X$ such that $f\left(x_{0}\right)=0$ and $f(x)=1$. Set $R:=x \otimes f$, and note 
that $R x_{0}=0$ and that $R^{*}\left(A^{-1} y_{0}\right)=f$ and $R^{*} f=f$. Thus

$$
\sigma_{R}\left(x_{0}\right)=\{0\} \text { and } \sigma_{R^{*}}\left(A^{-1} y_{0}\right)=\sigma_{R^{*}}\left(R^{*} A^{-1} y_{0}\right)=\sigma_{R^{*}}(f)=\{1\} .
$$

This contradiction finishes the proof.

3. Local spectral identity principle. This section is devoted to some local spectral identity principles, which we believe to be of interest in their own right. The first one characterizes in term of the local spectrum when two operators are linearly dependent.

THeOREM 3.1. Let $x_{0} \in X$ be a nonzero vector, and let $A, B \in \mathcal{B}(X)$. The following statements are equivalent.

(a) $A=\alpha B$ for some nonzero scalar $\alpha \in \mathbb{C}$.

(b) $\sigma_{A T}\left(x_{0}\right)=\{0\} \Longleftrightarrow \sigma_{B T}\left(x_{0}\right)=\{0\}$ for all operators $T \in \mathcal{B}(X)$.

(c) $\sigma_{A T}\left(x_{0}\right)=\{0\} \Longleftrightarrow \sigma_{B T}\left(x_{0}\right)=\{0\}$ for all rank one operators $T \in \mathcal{B}(X)$.

(d) $\sigma_{A T}^{*}\left(x_{0}\right)=\{0\} \Longleftrightarrow \sigma_{B T}^{*}\left(x_{0}\right)=\{0\}$ for all rank one operators $T \in \mathcal{B}(X)$.

Proof. We only need to show that $(d) \Rightarrow(a)$.

Assume that $\sigma_{A T}^{*}\left(x_{0}\right)=\{0\} \Longleftrightarrow \sigma_{B T}^{*}\left(x_{0}\right)=\{0\}$ for all rank one operators $T \in$ $\mathcal{B}(X)$, and let us first show that $A x_{0}$ and $B x_{0}$ are linearly dependent. Suppose to the contrary that $A x_{0}$ and $B x_{0}$ are linearly independent, and let us show that $x_{0}$, $A x_{0}$ and $B x_{0}$ are linearly dependent. If not, let $f_{0} \in X^{*}$ be a linear functional such that $f_{0}\left(x_{0}\right)=f_{0}\left(A x_{0}\right)=1$ and $f_{0}\left(B x_{0}\right)=0$. For $T_{0}:=x_{0} \otimes f_{0}$, we have $A T_{0}=A x_{0} \otimes f_{0}$ and $\sigma_{A T_{0}}\left(x_{0}\right)=\sigma_{A T_{0}}\left(x_{0}-A x_{0}\right) \cup \sigma_{A T_{0}}\left(A x_{0}\right)=\{0,1\}$. Moreover, $B T_{0}=B x_{0} \otimes f_{0}$ is a nilpotent rank one operator, and $\sigma_{B T_{0}}\left(x_{0}\right)=\{0\}$. Thus $\sigma_{A T_{0}}^{*}\left(x_{0}\right)=\{1\} \neq\{0\}=$ $\sigma_{B T_{0}}^{*}\left(x_{0}\right)$, and this contradiction shows that $x_{0}=\alpha A x_{0}+\beta B x_{0}$ for some scalars $\alpha, \beta \in \mathbb{C}$. Note that either $\alpha \neq 0$ or $\beta \neq 0$ and thus we may and shall assume that $\alpha \neq 0$. Let $f_{1} \in X^{*}$ be a linear functional such that $f_{1}\left(A x_{0}\right)=1$ and $f_{1}\left(B x_{0}\right)=0$. For $T_{1}:=x_{0} \otimes f_{1}$, we have $A T_{1}=A x_{0} \otimes f_{1}$ and $\{1\} \subset \sigma_{A T_{1}}\left(A x_{0}\right) \cup \sigma_{A T_{1}}\left(\beta B x_{0}\right) \subset\{0,1\}$, and thus $\sigma_{A T_{1}}^{*}\left(x_{0}\right)=\{1\}$. Note that $B T_{1}=B x_{0} \otimes f_{1}$ is a nilpotent rank one operator and $\sigma_{B T_{1}}\left(x_{0}\right)=\{0\}$. This shows that $\sigma_{A T_{1}}^{*}\left(x_{0}\right)=\{1\} \neq\{0\}=\sigma_{B T_{1}}^{*}\left(x_{0}\right)$, and yields to a contradiction. Thus, $A x_{0}=\alpha_{x_{0}} B x_{0}$ for some nonzero scalar $\alpha_{x_{0}}$; as desired.

Now, let $x$ be an arbitrary vector in $X$, and let $S \in \mathcal{B}(X)$ be an operator such that $S x_{0}=x$. Replacing $T$ by $S T$ in the statement (d), we note that $\sigma_{A S T}^{*}\left(x_{0}\right)=\{0\} \Longleftrightarrow$ $\sigma_{B S T}^{*}\left(x_{0}\right)=\{0\}$ for all rank one operators $T \in \mathcal{B}(X)$. By what has been shown above, there is $\alpha_{x}$ that $A x=A S x_{0}=\alpha_{x} B S x_{0}=\alpha_{x} B x$ for some nonzero scalar $\alpha_{x}$. Thus, there is a nonzero scalar $\alpha \in \mathbb{C}$ such that $A=\alpha B$; as desired.

The second result characterizes in term of the local spectrum when two operators are the same.

TheOREM 3.2. Let $x_{0} \in X$ be a nonzero vector, and let $A, B \in \mathcal{B}(X)$. The following statements are equivalent.

(a) $A=B$.

(b) $\sigma_{A T}\left(x_{0}\right)=\sigma_{B T}\left(x_{0}\right)$ for all operators $T \in \mathcal{B}(X)$.

(c) $\sigma_{A T}\left(x_{0}\right)=\sigma_{B T}\left(x_{0}\right)$ for all rank one operators $T \in \mathcal{B}(X)$.

(d) $\sigma_{A T}^{*}\left(x_{0}\right)=\sigma_{B T}^{*}\left(x_{0}\right)$ for all rank one operators $T \in \mathcal{B}(X)$.

Proof. We only need to show that $(d) \Rightarrow(a)$. So, assume that $\sigma_{A T}^{*}\left(x_{0}\right)=\sigma_{B T}^{*}\left(x_{0}\right)$ for all rank one operators $T \in \mathcal{B}(X)$, and note that there is a nonzero $\alpha \in \mathbb{C}$ such 
that $A=\alpha B$, by Theorem 3.1. To show that such $\alpha$ must be one, assume first that there is $x \in X$ such that $B x$ and $x_{0}$ are linearly independent, and let $f \in X^{*}$ be a linear functional such that $f(B x)=f\left(x_{0}\right)=1$. Set $T:=x \otimes f$, and note that $B T=$ $B x \otimes f$ and thus $\sigma_{B T}\left(x_{0}\right)=\sigma_{B T}\left(x_{0}-B x\right) \cup \sigma_{B T}(B x)=\{0,1\}$. It follows that $\{0,1\}=$ $\sigma_{B T}\left(x_{0}\right)=\sigma_{A T}\left(x_{0}\right)=\sigma_{\alpha B T}\left(x_{0}\right)=\{0, \alpha\}$, and $\alpha=1$.

Now, if $B x$ and $x_{0}$ are linearly dependent for any $x \in X$, then either $B=0$ and there is nothing to prove, or $B=x_{0} \otimes f$ for some $f \in X^{*}$. If the last case occurs, pick up $x \notin \operatorname{ker}(f)$ such that $f(x)=1$ and a linear functional $g \in X^{*}$ such that $g\left(x_{0}\right)=1$. Let $T:=x \otimes g$, and note that $B x=x_{0}$ and that $B T x_{0}=B x=x_{0}$. It follows that $\{1\}=\sigma_{B T}\left(x_{0}\right)=\sigma_{A T}\left(x_{0}\right)=\sigma_{\alpha B T}\left(x_{0}\right)=\{\alpha\}$.

4. Local spectral characterization of rank one operators. The following theorem, which may be of independent interest, gives a spectral characterization of rank one operators in term of local spectrum.

THEOREM 4.1. For a nonzero vector $x_{0}$ of $X$ and a nonzero operator $R \in \mathcal{B}(X)$, the following statements are equivalent.

(a) $R$ has rank one.

(b) $\sigma_{R T}^{*}\left(x_{0}\right)$ contains at most one element for all $T \in \mathcal{B}(X)$.

(c) $\sigma_{R T}^{*}\left(x_{0}\right)$ contains at most one element for all $T \in \mathcal{F}_{2}(X)$.

Proof. Clearly $(a) \Rightarrow(b) \Rightarrow(c)$, and so we only need to establish the implication $(c) \Rightarrow(a)$. So suppose that $\sigma_{R T}^{*}\left(x_{0}\right)$ contains at most one element for all $T \in \mathcal{F}_{2}(X)$, and assume for the sake of contradiction that $\operatorname{rank}(R) \geq 2$. Let us first show that $x_{0}$ is not in the range of $R$. Assume to the contrary that $x_{0}$ is in the range of $R$ so that there are $x, y \in X$ such that $x_{0}=R x$ and $v=R y$ are linearly independent. Set

$$
x_{1}=\frac{x-y}{2} \quad \text { and } \quad x_{2}=\frac{x+y}{2}
$$

and note that $x_{0}=R x_{1}+R x_{2}$. Now, choose two linear functionals $f_{1}$ and $f_{2}$ in $X^{*}$ such that $f_{1}\left(x_{0}\right)=1$ and $f_{1}(v)=-1$, and $f_{2}\left(x_{0}\right)=f_{2}(v)=2$ and thus $f_{1}\left(R x_{1}\right)=1, f_{1}\left(R x_{2}\right)=$ $0, f_{2}\left(R x_{1}\right)=0$ and $f_{2}\left(R x_{2}\right)=2$. For $S:=x_{1} \otimes f_{1}+x_{2} \otimes f_{2}$, we have $R S=\left(R x_{1}\right) \otimes$ $f_{1}+\left(R x_{2}\right) \otimes f_{2}$, and $R S\left(R x_{1}\right)=R x_{1}$ and $R S\left(R x_{2}\right)=2 R x_{2}$. As $x_{0}=R x_{1}+R x_{2}$, we have $\sigma_{R S}\left(x_{0}\right)=\sigma_{R S}\left(R x_{1}+R x_{2}\right)=\sigma_{R S}\left(R x_{1}\right) \cup \sigma_{R S}\left(R x_{2}\right)=\{1,2\}$. This contradiction shows $x_{0}$ is not in the range of $R$.

Now, let $u=R x$ and $v=R y$ from the range of $R$ so that $x_{0}, u$ and $v$ are linearly independent. Note that, since $u$ and $v$ are linearly independent, we see that either $x_{0}$ and $x$ or $x_{0}$ and $y$ are linearly independent. Suppose for instance that $x_{0}$ and $y$ are linearly independent, and choose two linear functionals $f$ and $g$ in $X^{*}$ such that $f\left(x_{0}\right)=1$ and $f(y)=0$, and $g\left(x_{0}\right)=0$ and $g(y)=1$. For $R_{1}:=x \otimes f+y \otimes g$, we have

$$
R R_{1} x_{0}=R x=u \quad \text { and } \quad R R_{1} y=R y=v .
$$

Set

$$
x_{3}:=\frac{x_{0}-y}{2} \quad \text { and } \quad x_{4}:=\frac{x_{0}+y}{2},
$$


and note that, since $x_{0}=x_{3}+x_{4}$ is not in the range of $R$, the two sets $\left\{x_{3}, R R_{1} x_{3}\right\}$ and $\left\{x_{4}, R R_{1} x_{4}\right\}$ cannot be simultaneously linearly dependent. Now, we shall discuss two cases.

Case 1. One of the two sets is linearly dependent and the other one is linearly independent. Suppose, for instant, that $R R_{1} x_{3}=\alpha x_{3}$ for some scalar $\alpha \in \mathbb{C}$. On the one hand, we note that, since $u$ and $v$ are linearly independent, such $\alpha$ which must be a nonzero scalar. On the other hand, $\left\{x_{3}, x_{4}, R R_{1} x_{4}\right\}$ must be linearly independent. If not, since $x_{3}$ and $x_{4}$ are linearly independent, there scalars $\alpha_{1}, \beta_{1} \in \mathbb{C}$ such that $R R_{1} x_{4}=\alpha_{1} x_{3}+\beta_{1} x_{4}$. Note that, since $R R_{1} x_{3}$ and $R R_{1} x_{4}$ are linearly independent, $\beta_{1} \neq 0$ and $R\left(x_{4}+\left(\beta_{1}-\alpha_{1}\right) \alpha^{-1} x_{3}\right)=\beta_{1}\left(x_{3}+x_{4}\right)=\beta_{1} x_{0}$. This contradicts the assumption that $x_{0}$ is not in the range of $R$, and shows that $x_{3}, x_{4}$ and $R R_{1} x_{4}$ are linearly independent. Now, choose two linear functionals $f_{3}$ and $f_{4}$ in $X^{*}$ such that $f_{3}\left(x_{3}\right)=1, f_{3}\left(x_{4}\right)=f_{3}\left(R R_{1} x_{4}\right)=0, f_{4}\left(x_{3}\right)=0$, and $f_{4}\left(x_{4}\right)=f_{4}\left(R R_{1} x_{4}\right)=2 \alpha$. For $T:=x_{3} \otimes f_{3}+x_{4} \otimes f_{4}$, we have $R R_{1} T=\left(R R_{1} x_{3}\right) \otimes f_{3}+\left(R R_{1} x_{4}\right) \otimes f_{4}$ and $R R_{1} T x_{3}=$ $R R_{1} x_{3}=\alpha x_{3}$, and

$$
R R_{1} T x_{4}=2 \alpha R R_{1} x_{4}, \quad \text { and } \quad R R_{1} T\left(R R_{1} x_{4}\right)=2 \alpha R R_{1} x_{4} .
$$

Thus, $\sigma_{R R_{1} T}\left(x_{3}\right)=\{\alpha\}$ and $\{2 \alpha\} \subset \sigma_{R R_{1} T}\left(x_{4}\right) \subset\{0,2 \alpha\}$. From this, it follows that $\{\alpha, 2 \alpha\} \subset \sigma_{R R_{1} T}\left(x_{0}\right)=\sigma_{R R_{1} T}\left(x_{3}\right) \cup \sigma_{R R_{1} T}\left(x_{4}\right) \subset\{0, \alpha, 2 \alpha\}$, and $\sigma_{R R_{1} T}^{*}\left(x_{0}\right)=\{\alpha, 2 \alpha\}$. This gives a contradiction and shows that $R$ must have rank one.

Case 2. The two sets $\left\{x_{3}, R R_{1} x_{3}\right\}$ and $\left\{x_{4}, R R_{1} x_{4}\right\}$ are linearly independent. If $\left\{x_{3}, R R_{1} x_{3}, x_{4}, R R_{1} x_{4}\right\}$ is linearly independent, then choose two linear functionals $g_{1}$ and $g_{2}$ in $X^{*}$ such that $g_{1}\left(x_{3}\right)=g_{1}\left(R R_{1} x_{3}\right)=1, g_{1}\left(x_{4}\right)=g_{1}\left(R R_{1} x_{4}\right)=$ $0, g_{2}\left(x_{3}\right)=g_{2}\left(R R_{1} x_{3}\right)=0$, and $g_{2}\left(x_{4}\right)=f_{4}\left(R R_{1} x_{4}\right)=2$. For $T:=x_{3} \otimes g_{1}+x_{4} \otimes$ $g_{2}$, we have $R R_{1} T=\left(R R_{1} x_{3}\right) \otimes g_{1}+\left(R R_{1} x_{4}\right) \otimes g_{2}$. Then $R R_{1} T x_{3}=R R_{1} x_{3}$, and $R R_{1} T\left(R R_{1} x_{3}\right)=R R_{1} x_{3}$, and thus $\{1\} \subset \sigma_{R R_{1} T}\left(x_{3}\right) \subset\{0,1\}$. We also have $R R_{1} T x_{4}=$ $2 R R_{1} x_{4}$, and $R R_{1} T\left(R R_{1} x_{4}\right)=2 R R_{1} x_{4}$, and thus $\{2\} \subset \sigma_{R R_{1} T}\left(x_{4}\right) \subset\{0,2\}$. From

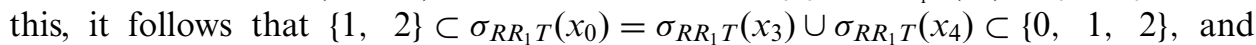
$\sigma_{R R_{1} T}^{*}\left(x_{0}\right)=\{1,2\}$. This contradiction shows that $\left\{x_{3}, R R_{1} x_{3}, x_{4}, R R_{1} x_{4}\right\}$ is linearly dependent.

Now, suppose that $x_{3}=\alpha x_{4}+\beta R R_{1} x_{3}+\gamma R R_{1} x_{4}$ for some scalars $\alpha, \beta, \gamma \in \mathbb{C}$, and pick up two linear functionals $g_{3}$ and $g_{4}$ in $X^{*}$ such that $g_{3}\left(R R_{1} x_{3}\right)=1, g_{3}\left(x_{4}\right)=$ $g_{3}\left(R R_{1} x_{4}\right)=0, g_{4}\left(R R_{1} x_{3}\right)=0, g_{4}\left(x_{4}\right)=-2 \gamma / \alpha$, and $g_{4}\left(R R_{1} x_{4}\right)=2$. For $P:=x_{3} \otimes$ $g_{3}+x_{4} \otimes g_{4}$, we have $R R_{1} P=\left(R R_{1} x_{3}\right) \otimes g_{3}+\left(R R_{1} x_{4}\right) \otimes g_{4}$ and $R R_{1} P x_{3}=\beta R R_{1} x_{3}$, and $R R_{1} P\left(R R_{1} x_{3}\right)=R R_{1} x_{3}$. Thus $\{1\} \subset \sigma_{R R_{1} P}\left(x_{3}\right) \subset\{0,1\}$. We also have $R R_{1} P x_{4}=$ $-\frac{2 \gamma}{\alpha} R R_{1} x_{4}$, and $R R_{1} P\left(R R_{1} x_{4}\right)=2 R R_{1} x_{4}$, and thus $\{2\} \subset \sigma_{R R_{1} P}\left(x_{4}\right) \subset\{0,2\}$. It follows that $\{1,2\} \subset \sigma_{R R_{1} T}\left(x_{0}\right)=\sigma_{R R_{1} T}\left(x_{3}\right) \cup \sigma_{R R_{1} T}\left(x_{4}\right) \subset\{0,1,2\}$, and $\sigma_{R R_{1} T}^{*}\left(x_{0}\right)=$ $\{1,2\}$. This contradiction shows that $R$ has rank one in this case too.

5. Proof of theorem 1.1. We only need to establish the 'only if' part whose proof breaks down into several steps. Assume that $\varphi$ satisfies (1.1), and let us first show that $\varphi$ is injective and $\varphi(0)=0$. If $\varphi(A)=\varphi(B)$ for some $A, B \in \mathcal{B}(X)$, we get

$$
\sigma_{A T}\left(x_{0}\right)=\sigma_{\varphi(A) \varphi(T)}\left(y_{0}\right)=\sigma_{\varphi(B) \varphi(T)}\left(y_{0}\right)=\sigma_{B T}\left(x_{0}\right)
$$

for all $T \in \mathcal{B}(X)$. By Theorem 3.2, we see that $A=B$ and $\varphi$ is injective. But since $\varphi$ is assumed to be surjective, the map $\varphi$ is, in fact, bijective. For the second part of the 
claim, note that for every $T \in \mathcal{B}(X)$, we have $\{0\}=\sigma_{0 \times T}\left(x_{0}\right)=\sigma_{\varphi(0) \varphi(T)}\left(y_{0}\right)$. Again by Theorem 3.2 and the bijectivity of $\varphi$, we see that $\varphi(0)=0$.

Second let us show that either

$$
\sigma_{\varphi(T)}\left(y_{0}\right)=\sigma_{T}\left(x_{0}\right)
$$

for all $T \in \mathcal{B}(X)$, or

$$
\sigma_{\varphi(T)}\left(y_{0}\right)=-\sigma_{T}\left(x_{0}\right)
$$

for all $T \in \mathcal{B}(X)$. To do so, we first prove that either $\varphi(\mathbf{1})=\mathbf{1}$ or $\varphi(\mathbf{1})=-\mathbf{1}$. Indeed, for every $T \in \mathcal{B}(X)$, we have $\{0\}=\sigma_{T}\left(x_{0}\right)=\sigma_{T^{2}}\left(x_{0}\right) \Longleftrightarrow\{0\}=\sigma_{\varphi(T)^{2}}\left(y_{0}\right)=\sigma_{\varphi(T)}\left(y_{0}\right)$, and thus $\sigma_{\varphi(T)}\left(y_{0}\right)=\{0\} \Longleftrightarrow \sigma_{T}\left(x_{0}\right)=\{0\}=\sigma_{1 T}\left(x_{0}\right) \Longleftrightarrow \sigma_{\varphi(\mathbf{1}) \varphi(T)}\left(y_{0}\right)=\{0\}$. By the surjectivity of $\varphi$ and Theorem 3.1, we see that $\varphi(\mathbf{1})=\alpha \mathbf{1}$ for some nonzero scalar $\alpha \in \mathbb{C}$. Such $\alpha$ must be 1 or -1 since $\{1\}=\sigma_{1^{2}}\left(x_{0}\right)=\sigma_{\varphi(\mathbf{1})^{2}}\left(y_{0}\right)=\sigma_{\alpha^{2} \mathbf{1}}\left(y_{0}\right)=\left\{\alpha^{2}\right\}$. If $\varphi(\mathbf{1})=\mathbf{1}$, then for every $T \in \mathcal{B}(X)$, we have

$$
\sigma_{T}\left(x_{0}\right)=\sigma_{1 \times T}\left(x_{0}\right)=\sigma_{\varphi(\mathbf{1}) \varphi(T)}\left(y_{0}\right)=\sigma_{\mathbf{1} \times \varphi(T)}\left(y_{0}\right)=\sigma_{\varphi(T)}\left(y_{0}\right),
$$

and (5.3) is established. If $\varphi(\mathbf{1})=-\mathbf{1}$, then

$$
\sigma_{T}\left(x_{0}\right)=\sigma_{\mathbf{1} \times T}\left(x_{0}\right)=\sigma_{\varphi(\mathbf{1}) \varphi(T)}\left(y_{0}\right)=\sigma_{-\mathbf{1} \times \varphi(T)}\left(y_{0}\right)=-\sigma_{\varphi(T)}\left(y_{0}\right)
$$

for all $T \in \mathcal{B}(X)$. This establish (5.4).

Now, we show that $\varphi$ is a linear map preserving rank one operators in both directions. Let $R \in \mathcal{B}(X)$ be a rank one operator, and note that, since $\varphi(0)=0$ and $\varphi$ is bijective, $\varphi(R) \neq 0$. Moreover, for every operator $S=\varphi(T) \in \mathcal{B}(Y)$, we have $\sigma_{R T}^{*}\left(x_{0}\right)=$ $\sigma_{\varphi(R) \varphi(T)}^{*}\left(y_{0}\right)=\sigma_{\varphi(R) S}^{*}\left(y_{0}\right)$ contains at most one element. By Theorem 4.1, we see that $\varphi(R)$ has rank one. The converse holds in a similar way and thus $\varphi$ preserves the rank one operators in both directions; as desired.

To establish the linearity of $\varphi$, let us first show that $\varphi$ is homogenous. For every $\lambda \in \mathbb{C}$ and $A, T \in \mathcal{B}(X)$, we have

$$
\sigma_{\lambda \varphi(A) \varphi(T)}\left(y_{0}\right)=\lambda \sigma_{\varphi(A) \varphi(T)}\left(y_{0}\right)=\lambda \sigma_{A T}\left(x_{0}\right)=\sigma_{\lambda A T}\left(x_{0}\right)=\sigma_{\varphi(\lambda A) \varphi(T)}\left(y_{0}\right) .
$$

Since $\varphi$ is surjective, Theorem 3.2 shows that $\varphi(\lambda A)=\lambda \varphi(A)$; as desired. Now, let us show that $\varphi$ is additive. Note that, by Theorem 4.1, the map $\varphi$ preserves rank one operators in both directions. Let $R \in \mathcal{B}(X)$ be a rank one operator and $T, S \in \mathcal{B}(X)$, and note that, by Lemma 2.2, we have

$$
\begin{aligned}
\sigma_{\varphi(T+S) \varphi(R)}^{*}\left(y_{0}\right) & =\sigma_{(T+S) R}^{*}\left(x_{0}\right)=\sigma_{T R}^{*}\left(x_{0}\right)+\sigma_{S R}^{*}\left(x_{0}\right) \\
& =\sigma_{\varphi(T) \varphi(R)}^{*}\left(y_{0}\right)+\sigma_{\varphi(S) \varphi(R)}^{*}\left(y_{0}\right) \\
& =\sigma_{(\varphi(T)+\varphi(S)) \varphi(R)}^{*}\left(y_{0}\right) .
\end{aligned}
$$

By the arbitrariness of the rank one operator $R$, the bijectivity of $\varphi$ and Theorem 3.1, we deduce that

$$
\varphi(T+S)=\varphi(T)+\varphi(S)
$$

for all $T, S \in \mathcal{B}(X)$, and $\varphi$ is linear. 
Finally, let us show that $\varphi$ takes the desired form. Since $\varphi$ is a bijective linear map preserving the rank one operators in both directions, either there are bijective linear mappings $A: X \rightarrow Y$ and $B: X^{*} \rightarrow Y^{*}$ such that

$$
\varphi(x \otimes f)=A x \otimes B f,\left(x \in X, f \in X^{*}\right),
$$

or there are bijective linear mappings $A: X^{*} \rightarrow Y$ and $B: X \rightarrow Y^{*}$ such that

$$
\varphi(x \otimes f)=A f \otimes B x,\left(x \in X, f \in X^{*}\right) ;
$$

see for instance [16]. By Lemma 2.4, the second form cannot occur, and thus $\varphi$ takes the form (5.5). Since $\varphi$ satisfies either (5.3) or (5.4), Lemma 2.3, shows that $A$ is a continuous map for which $A x_{0}=\alpha y_{0}$ for some nonzero scalar $\alpha \in \mathbb{C}$, and $\varphi(x \otimes f)=$ $\pm A(x \otimes f) A^{-1}$ for all $x \in X$ and $f \in X^{*}$. Replacing $A$ by $\alpha^{-1} A$, we may and shall assume that $A x_{0}=y_{0}$. To finishes the proof, note that for every rank one operator $R \in \mathcal{B}(X)$ and every $T \in \mathcal{B}(X)$, we have

$$
\sigma_{ \pm A T A^{-1} \varphi(R)}\left(y_{0}\right)=\sigma_{A T A^{-1} A R A^{-1}}\left(y_{0}\right)=\sigma_{A T R A^{-1}}\left(y_{0}\right)=\sigma_{T R}\left(x_{0}\right)=\sigma_{\varphi(T) \varphi(R)}\left(y_{0}\right) .
$$

By Theorem 3.2, we see that $\varphi(T)= \pm A T A^{-1}$ for all $T \in \mathcal{B}(X)$. The proof is now complete.

Acknowledgement. The restriction to infinite-dimensional Banach spaces in the statement of our main result is just for the sake of simplicity. This work was supported by NSERC (Canada) and FQRNT (Québec).

\section{REFERENCES}

1. P. Aiena, Fredholm and local spectral theory, with applications to multipliers (Kluwer, Dordrecht, 2004).

2. R. Bhatia, P. Šemrl and A. Sourour, Maps on matrices that preserve the spectral radius distance, Stud. Math. 134(2) (1999), 99-110.

3. A. Bourhim and J. Mashreghi, Local spectral radius preservers, Integral Equations Operator Theory 76(1) (2013), 95-104.

4. A. Bourhim and J. Mashreghi, Maps preserving the local spectrum of triple product of operators, Linear Multilinear Algebra, to appear.

5. A. Bourhim and T. Ransford, Additive maps preserving local spectrum, Integral Equations Operator Theory 55 (2006) 377-385.

6. J. Bračič and V. Müller, Local spectrum and local spectral radius of an operator at a fixed vector, Stud. Math. 194(2) (2009), 155-162.

7. J. T. Chan, C. K. Li and N. S. Sze, Mappings preserving spectra of products of matrices, Proc. Am. Math. Soc. 135 (2007), 977-986.

8. C. Costara, Linear maps preserving operators of local spectral radius zero, Integral Eqns Operator Theory 73(1) (2012), 7-16.

9. C. Costara, Automatic continuity for linear surjective mappings decreasing the local spectral radius at some fixed vector, Arch. Math. 95(6) (2010), 567-573.

10. J. L. Cui and J. C. Hou, Maps leaving functional values of operator products invariant, Linear Algebra Appl. 428 (2008), 1649-1663.

11. J. C. Hou and Q. H. Di, Maps preserving numerical range of operator products, Proc. Am. Math. Soc. 134 (2006), 1435-1446.

12. A. A. Jafarian and A. R. Sourour, Spectrum-preserving linear maps, J. Funct. Anal. 66 (1986), 255-261. 
13. K. B. Laursen and M. M. Neumann, An introduction to local spectral theory, London Mathematical Society Monograph, New Series, vol. 20, 2000.

14. C. K. Li, P. Šemrl and N. S. Sze, Maps preserving the nilpotency of products of operators, Linear Algebra Appl. 424 (2007), 222-239.

15. L. Molnár, Some characterizations of the automorphisms of $B(H)$ and $C(X)$, Proc. Am. Math. Soc. 130 (2002), 111-120.

16. M. Omladič and P. Šemrl, Additive mappings preserving operators of rank one, Linear Algebra Appl. 182 (1993), 239-256.

17. M. Wang, L. Fang and G. Ji, Linear maps preserving idempotency of products or triple Jordan products of operators, Linear Algebra Appl. 429 (2008), 181-189. 